\title{
Soap-Free Latices of Polyoxyethylene Chain-Binding Particles
}

\author{
Futoshi Hoshino, Mami SaKaI, Haruma KaWAGUCHI, \\ and Yasuji OHTSUKA \\ Department of Applied Chemistry, Faculty of Science \& Technology, \\ Keio University, 3-14-1 Hiyoshi, Kohoku-ku, Yokohama 223, Japan
}

(Received October 22, 1986)

\begin{abstract}
Four kinds of oxyethylene methacrylates having different oxyethylene chain lengths were copolymerized with styrene in soap-free media. Copolymerization using longer oxyethylene chain-carrying monomer formed smoother and smaller, but highly hydrated latex particles. Stability of latices also depended on the oxyethylene chain length. The stability of latices composed of long-chain oxyethylene methacrylates changed with temperature and they showed reversible transition between dispersion and flocculation by changing temperature.

KEY WORDS Soap-Free Emulsion Polymerization / Latex / Polyoxyethylene Chain / Hydration-Dehydration / Stability / Flocculation /
\end{abstract}

Latex particles covered by groups capable of forming hydrogen-bonds weakly or moderately lose their dispersion-stability above a specific temperature and a specific electrolyte concentration but recover it reversibly by decreasing temperature. The transition is due to reversible hydration and dehydration of hydrophilic groups with changing temperature and/or electrolyte concentration as shown in Figure 1. Some $N$-substituted acrylamides are typical examples for such groups and their specific properties were studied in our previous paper. ${ }^{1}$ Another candidate of such groups whose extent of hydration is sensitive to temperature is oxyethylene chain. Polyoxyethylene-type surfactants have their characteristic cloud points dependent on the hydrophileliophile balance or the length of oxyethylene chain. Therefore, it was expected that oxyethylene methacrylate (OM) would form latex particles whose stability depends on the oxyethylene chain length. In this study styrene was copolymerized with four kinds of OMs having oxyethylene chains of different length in emulsifier-free aqueous media and the de- pendence of the properties of resulting latices on the kind of OMs was investigated.

\section{EXPERIMENTAL}

\section{Materials}

The four kinds of OMs employed in this study are shown in Table I.

The last three OMs in Table I were given by Nippon Oil \& Fats Co., Ltd. (Blenmer series). HEMA, PE-90 and styrene (St) were distilled at $68^{\circ} \mathrm{C} / 1 \mathrm{mmHg} 76^{\circ} \mathrm{C} / 0.2 \mathrm{mmHg}$, and $46^{\circ} \mathrm{C} /$ $21.5 \mathrm{mmHg}$, respectively. Other monomers, PE-200 and PE-350, and an initiator, 2,2'azobis(2-amidinopropane)dichloride (Wako Chemicals Co., V-50), were used as received. Another initiator, potassium persulfate was recrystallized from water.

\section{Polymerization}

Soap-free copolymerization of St with OMs was carried out in a $200 \mathrm{ml}$ four-neck roundbottom flask equipped with stirrer, nitrogen inlet, condenser, and serum rubber. Standard recipe for the copolymerization is as follows: 


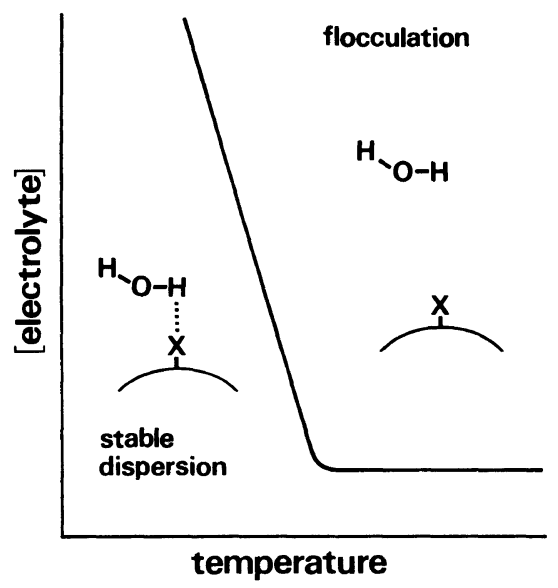

Figure 1. Reversible dispersion-flocculation of latex as a function of electrolyte concentration and temperature.

$(\mathrm{St}+\mathrm{OM}) 20 \mathrm{~g}$, distilled water $120 \mathrm{~g}, \mathrm{~V} 500.2 \mathrm{~g}$. The weight fraction of $\mathrm{OM}$ in monomer feed $(f)$ was varied from 0.05 to 0.2 . The polymerizations were carried out for $24 \mathrm{~h}$ at the prescribed temperature.

\section{Characterization}

Total conversion and the amount of the polymer dissolving in aqueous phase was determined by gravimetry. The size of dry particles was measured on electron micrographs. Hydrodynamic size was determined using a sub-micron particle analyzer Coulter N4. The minimum concentration of $\mathrm{NaCl}$ to flocculate a latex was defined as the critical flocculation concentration and determined at several temperature ranging from 15 to $70^{\circ} \mathrm{C}^{2}$ Electrophoretic mobility of particles was measured using an electrophoresis microscope system of Sugiura Lab. Co. The surface charge of particles was determined by conductometric titration of ion-exchanged latices.

\section{RESULTS AND DISCUSSION}

\section{Shape of Latex Particles}

Polymerization using OMs with short oxyethylene chains (S-OM) generally resulted in the formation of uneven-shaped particles. On
Table I. Oxyethylene-type methacrylates used

\begin{tabular}{|c|c|}
\hline$n$ & Abbreviation \\
\hline 1 & HEMA \\
\hline 2 & PE-90 \\
\hline $4-5$ & PE-200 \\
\hline $7-8$ & PE-350 \\
\hline
\end{tabular}

the other hand, OM with long oxyethylene chains (L-OM) served to form smooth and spherical copolymer particles. Typical results are shown in Figure 2. These results would be attributed to the different timing of polymerization between hydrophilic and hydrophobic monomers, ${ }^{3}$ in our case, OM and St and to the different modes of phase separation of OM-rich polymer with St-rich polymer in each particle. It is supposed that, in the system of S-OMs, OM units-rich domains disperse in the poly-St matrix and are responsible for the uneven shape of particles. On the contrary, L-OM-rich polymers, which are more hydrophilic and more flexible, would diffuse to the surface of particle during phase separation, forming a surface layer or skin.

The shapes of particles were also affected by the $f$ values and the concentration of initiator as shown in Figure 2. It is probable that the increase in $f$ made phase-separated domains larger and, as a result, enhanced the unevenness of particles. In some literature dealing with the effects of the initiator concentration on particle shape, it is mentioned that frequent entry of radicals into growing particles from water phase due to the high initiator concentration makes more domains at many sites in particles before their diffusion and, consequently, contribute to the formation of uneven-shaped particles. ${ }^{4-6}$ The results in Figure 2 indicate the same tendency. 


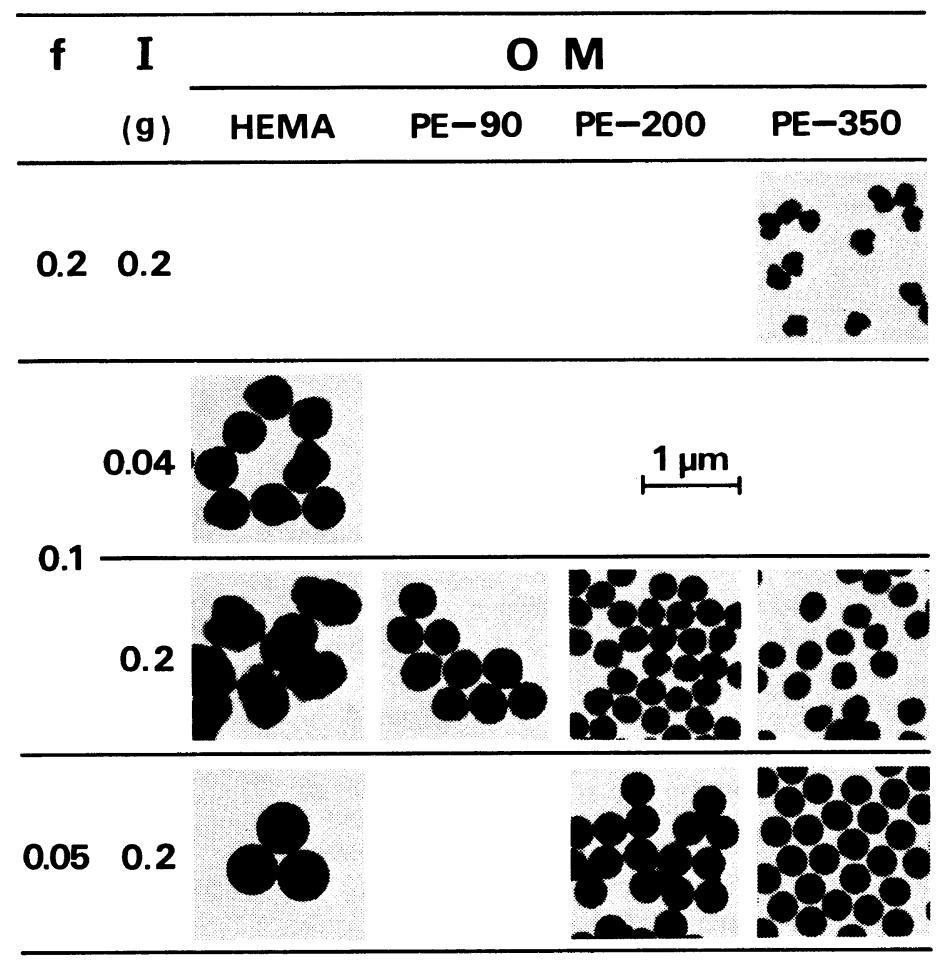

Figure 2. Transmission electron micrographs of latex particles prepared under different conditions. Recipe, monomer-water-V50 $=20: 120:(0.04$ or 0.20$)(\mathrm{g})$; polymerization temperature, $60^{\circ} \mathrm{C}$.

Table II. Particle size measured by different methods

\begin{tabular}{lcccc}
\hline & \multicolumn{4}{c}{ Average diameter/nm } \\
\cline { 2 - 5 } Instrument used & HEMA/St & PE-90 $\times$ St & PE-200/St & PE-350/St \\
\hline Electron microscope & 425 & 320 & 302 & 277 \\
Sub-micron analyzer at $30^{\circ} \mathrm{C}$ & - & 357 & 381 & 420 \\
\hline
\end{tabular}

Latex preparation: St-methacrylate-water-V $50=19: 1: 120: 0.2(\mathrm{~g}), 60^{\circ} \mathrm{C}$.

\section{Size of Latex Particles}

The size of latex particles composed of $\mathrm{St}$ and OMs depended on the kind of OMs. Some data on particle size are shown in Table II. Electron micrographs of dry particles indicated the copolymerization of St with L-OM formed smaller particles but, hydrodynamic measurements showed an inverse trend with respect to the effects of oxyethylene chain length on the particle size in dispersion. The increase in hydrodynamic size of L-OM- containing particles cannot be attributed to any aggregation because such particles are obviously dispersing more stably than S-OMcontaining particles.

To discuss the above-mentioned effects of oxyethylene chain length on particle size, the characteristics of a series of particles prepared at $f=0.05$ were examined. The ratio of $\mathrm{OM}$ existing in or on the particles to fed ones was measured and the results are shown in Table IIIa. More hydrophilic L-OMs are apt to re- 
Table III. Distribution of methacrylate units in lattices ${ }^{\mathrm{a}}$

a. Particle-forming methacrylate/fed methacrylate

\begin{tabular}{lcccc}
\hline$f$ & HEMA/St & PE-90/St & PE-200/St & PE-350/St \\
\hline 0.05 & 0.74 & 0.73 & 0.56 & 0.36 \\
0.1 & 0.87 & 0.76 & 0.49 & 0.37 \\
\hline
\end{tabular}

b. Apparent surface density of methacrylate units $/ \mathrm{nm}^{2}$

\begin{tabular}{lcccc}
\hline$f$ & HEMA/St & PE-90/St & PE-200/St & PE-350/St \\
\hline 0.05 & 14.6 & 8.5 & 3.3 & 1.3 \\
0.1 & 42.7 & 19.3 & 5.8 & 2.9 \\
\hline
\end{tabular}

a Latex preparation: ( $\mathrm{St}+$ methacrylate)-watar-V50= $20: 120: 0.2(\mathrm{~g}), 60^{\circ} \mathrm{C}$.

main more in the aqueous medium as expected. Then the apparent surface density of OM units was calculated by assuming that all the $\mathrm{OM}$ units are located in the thin surface layer (Table IIIb). The apparent surface density of OMs decreased with increasing oxyethylene chain length. The very large apparent density of HEMA units perhaps implies that the above-mentioned assumption is incorrect for HEMA-containing particles. Namely, it is likely that HEMA units would not be located in the surface layer but rather distribute even inside particles. The specific surface area, reciprocal of apparent surface density, of a PE350 unit was close to that of an analogous nonionic surfactant. The results in Tables II and III indicate that a lesser amount of L-OMs can stabilize a larger surface area to form a larger number of smaller particles. It might be that the difference between hydrodynamic diameter and diameter measured by electron micrographs is twice the thickness of the hydrated layer constructed by oxyethylene chains of on-surface OMs. But trial calculation proved that the speculation is questionable because the calculated degree of hydration per oxyethylene unit was extraordinarily large. In this sense, what the absolute values of hydrodynamic size measured by sub-micron particle ana-

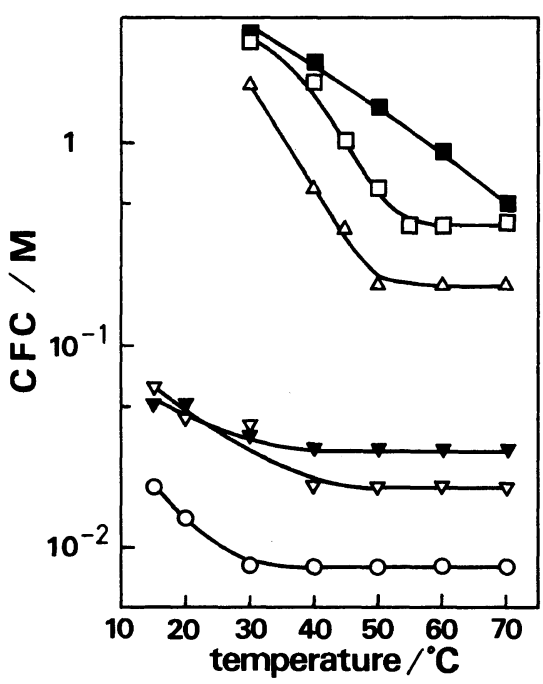

Figure 3. Effects of temperature on the stability of latices in $\mathrm{NaCl}$ aqueous solution. $\bigcirc$, HEMA/St; $\nabla, \nabla$, PE-90/St; $\triangle$, PE-200/St; $\square$, $\square$, PE-350/St. Open symbols, V50 initiation; closed symbols, KPS initiation; latex preparation, $\mathrm{St}$-methacrylate-water-initiator $=$ $18: 2: 120: 0.2(\mathrm{~g})$; polymerization temperature, $60^{\circ} \mathrm{C}$.

lyzer mean is obscure in our present system. However, the values would be meaningful if they are compared with each other and longer oxyethylene chains are believed to promote the stabilization of particles by the construction of the thicker hydrated layer. A comparison of stability among the latices composed of different OMs is made in the next section.

In typical emulsion polymerization, the rate of polymerization is proportional to the number of particles. This was not the case in our system. The discrepancy would result from contamination with uneliminable inhibitors in PE-200 and 350.

\section{Stability of Latices}

The curves which distinguish between stable and unstable ranges of latices with respect to temperature and electrolyte concentration were obtained by CFC measurement and the results are shown in Figure 3. Every curve is composed of two parts, a descending part with increasing temperature and a horizontal part. 
The inflection point is referred to as the critical flocculation temperature (CFT). The gradual decrease in stability with increasing temperature up to CFT is attributed to a thermal disturbance of hydrogen bonds or dehydration of on-surface hydrophilic groups. The curves in Figure 3 indicate that longer oxyethylene chains are more effective for the stabilization of particles than shorter oxyethylene chains.

The CFTs of latices changed from about $30^{\circ} \mathrm{C}$ for $\mathrm{HEMA} / \mathrm{St}$ latex to $50^{\circ} \mathrm{C}$ for $\mathrm{PE} 350 / \mathrm{St}$ latex. The chain-length dependence of CFT resembles that of cloud points of polyoxyethylene-type nonionic surfactants. Once Napper investigated the CFT of latices stabilized by several poly(ethylene oxide)-containing block copolymers and reported that the CFTs are about $45^{\circ} \mathrm{C}$ independent of the chain length of poly(ethylene oxide) fragments. ${ }^{7}$ This value is close to that of $\mathrm{L}-\mathrm{OM} / \mathrm{St}$ latices. The cause for no dependence of CFT on the chain length in Napper's result would be the high degree of polymerization of poly(ethylene oxide). He used poly(ethylene oxide)s having a degree of polymerization more than 20. Somewhat high CFTs of L$\mathrm{OM} / \mathrm{St}$ latices compared with that of Napper's might be attributed to efficient combining of poly(ethylene oxide) chains on $\mathrm{OM} / \mathrm{St}$ particles.

It is reasonable to consider that, for the above CFT, the extent of hydration is unchanged at the minimum value and the stability of latices is governed by electrostatic and van der Waals forces. This was the case for $N$ substituted acrylamides/St copolymer latices; that is, the relation between dispersion stability and surface potential of the latex particles was qualitatively explainable on the basis of the DLVO theory. However, in OM/St latices a different dependence of stability on the surface potential was observed. The product of electrophoretic mobility with viscosity of the medium, a measure of surface potential, is plotted against temperature in Figure 4. The surface potential was low for L-OM/St particle

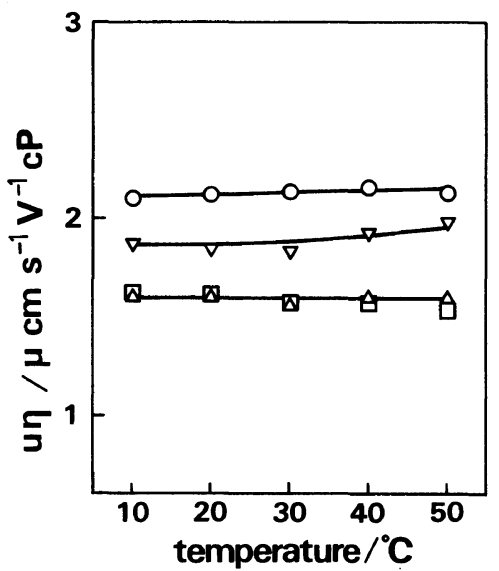

Figure 4. Product of electrophoretic mobility $(u)$ with viscosity $(\eta)$ as a function of temperature. $\mathrm{O}, \mathrm{HEMA} / \mathrm{St}$; $\nabla$, PE-90/St; $\triangle$, PE-200/St; $\square$, PE-350/St. NaCl concentration, $10^{-2} \mathrm{M}$; latex preparation, St-methacrylatewater-V50 = 18:2:120:0.2 (g); polymerization temperature, $60^{\circ} \mathrm{C}$.

Table IV. Temperature dependence of hydrodynamic size of PE-200/St particles ${ }^{\mathrm{a}}$

\begin{tabular}{cc}
\hline Temperature $/{ }^{\circ} \mathrm{C}$ & Hydrodynamic diameter $/ \mathrm{nm}$ \\
\hline 30 & 481 \\
40 & 461 \\
50 & 473 \\
60 & 456 \\
\hline \multicolumn{2}{c}{ Diameter measured on } \\
electron micrograph \\
\hline \multirow{2}{*}{ Latex preparation: St-methacrylate-water-KPS $=$} \\
$18: 2: 120: 0.2(\mathrm{~g}), 60^{\circ} \mathrm{C}$.
\end{tabular}

regardless of temperature range. Long oxyethylene chains seem to bury a part of ionic groups within themselves. According to the DLVO theory, the low surface potential and small size (at dry state) of L-OM/St particles are supposed to bring about an unstable latex. Nevertheless, L-OM/St latices are more stable than $\mathrm{S}-\mathrm{OM} / \mathrm{St}$ latices even above CFT as shown in Figure 3. This result is hard to explain. The following hypothesis might be. applicable; differing from $N$-substituted acrylamides/St systems, shrinkage of polyoxyethylene layer, accompanied with dehydra- 
Table V. Surface charge density of latices prepared by different initiators ${ }^{\mathrm{a}}$

\begin{tabular}{|c|c|c|c|c|c|}
\hline \multirow{2}{*}{ Initiator } & & \multicolumn{4}{|c|}{ Surface charge (initiator fragment) density } \\
\hline & & HEMA/St & $\mathrm{PE}-90 / \mathrm{St}$ & $\mathrm{PE}-200 / \mathrm{St}$ & PE-350/St \\
\hline \multirow{2}{*}{ V50 } & (eq/g particle) & 22.7 & 11.2 & 6.5 & 5.9 \\
\hline & (unit/nm $/ \mathrm{nm}^{2}$ ) & - & - & 0.14 & 0.13 \\
\hline \multirow[t]{2}{*}{ KPS } & (eq/g particle) & 33.3 & 19.5 & 11.3 & 11.7 \\
\hline & (unit $/ \mathrm{nm}^{2}$ ) & - & - & 0.39 & 0.33 \\
\hline
\end{tabular}

a Latex preparation: St-methacrylate-water-initiator $=18: 2: 120: 0.2(\mathrm{~g}), 60^{\circ} \mathrm{C}$.

tion by an increase in temperature, is not so drastic that, even above CFT, the actual particle size of $\mathrm{L}-\mathrm{OM} / \mathrm{St}$ particles in water is still large enough to keep high electrostatic repulsion (proportional to particle size) between particles. Little shrinkage of hydrophilic layer with increasing temperature is evident by temperature-insensitive curves in Figure 4. In fact the hydrodynamic size measured at various temperatures (Table IV) indicated no significant shrinking with increasing temperature. The bulky polyoxyethylene layer might make some contribution to the stabilization of dispersion with a slightly changed conformation even though it lost tightly-bound water above CFT.

\section{Effect of Different Initiator Species}

Two kinds of initiators were used in this study. V50 and KPS systems formed particles with similar shape. Their characteristics were compared and it was found that the surface charge density differed between the two initiator systems. The results are shown in Table V. The less surface density of particles of V50 systems is believed to result from the penetration of a larger number of V50 fragments into particles due to their lower polarity compared with that of KPS fragments. It was checked whether the difference in surface charge density between two systems affects the CFCs or not. The CFCs of two series of latices was approximately identical in the low temperature range. However, in the high temperature range, latices prepared by KPS were more stable than those of V50 system (Figure 3) possibly due to increased electrostatic effect after dehydration of nonionic hydrophilic layer on the particle.

\section{CONCLUSIONS}

Latices whose particles have bound polyoxyethylene chains were obtained by soapfree emulsion copolymerization of styrene with polyoxyethylene methacrylates. The size and shape of latex particles depended significantly on the oxyethylene chain length of comonomers as well as monomer composition and initiator concentration. Longer oxyethylene chain-having comonomers formed more stable latices and such latices showed temperaturesensitive stability having specific transition points. The kinds of initiator affected the stability of latices in high temperature range due to some differences in the polarity of the species.

\section{REFERENCES}

1. H. Kawaguchi, F. Hoshino, and Y. Ohtsuka, Makromol. Chem., Rapid Commun., 7, 109 (1986).

2. F. Hoshino, T. Fujimoto, H. Kawaguchi, and Y. Ohtsuka, Polym. J., 19, 241 (1987).

3. C. F. Zukoski and D. A. Saville, J. Colloid Interface Sci., 104, 583 (1985).

4. T. Matsumoto, M. Ohkubo, and S. Shibao, Kobunshi Ronbunshu, 33, 575 (1976).

5. S. Kamei, M. Ohkubo, and T. Matsumoto, Preprint, 3rd Polymer Microsphere Symposium, Fukui, 1984, 
Latices of Polyoxyethylene Chain-Binding Particles

p 53.

6. I. Cho and K.-W. Lee, J. Appl. Polym. Sci., 30, 1903 (1985).
7. D. H. Napper, J. Colloid Interface Sci., 32, 106 (1970). 